\title{
Antimicrobial Susceptibility Profile of $E$. coli Isolates Causing Urosepsis: Single Centre Experience
}

\author{
Pragya Agarwala ${ }^{1}$, Archa Sharma ${ }^{2} *$ and Kamal Sharma ${ }^{3}$ \\ ${ }^{1}$ Department of Microbiology, All India Institute of Medical Sciences, Raipur, India \\ ${ }^{2}$ Department of Microbiology, All India Institute of Medical Sciences, Bhopal, India \\ ${ }^{3}$ Department of Cardiology, U. N. Mehta Institute of Cardiology and Research Centre, \\ Ahmedabad, Gujarat, India, India
}

*Corresponding author

\begin{tabular}{|l|}
\hline Ke y w o r d s \\
Antimicrobial \\
Susceptibility, \\
Urosepsis \\
E. coli
\end{tabular}

A B S T R A C T
Urosepsis is defined as presence of systemic inflammatory response syndrome secondary to a severe infection of the urinary tract, with the risk of multi-organ dysfunction and shock. This study was designed to analyze the antimicrobial susceptibility profile of $E$. coli isolates causing urosepsis and to compare it with the susceptibility profile of other isolates of $E$. coli from blood stream infection. And to study the various factors associated with $E$. coli urosepsis cases. A total of 689 isolates of $E$. coli were isolated from blood cultures in 2015. Of them 112 were identified to be associated with urosepsis. The mean age of the urosepsis patients was 46.26 years (SD 17.24) and $65.1 \%$ were females. The prevalence of Extended spectrum beta lactamase production (ESBL), as screened by disc diffusion testing using ceftazidime was almost similar in the two groups, $76 \%$ in the isolates from urosepsis, and $72 \%$ in the other group ( $\mathrm{p}$ value $>0.05$ ). Carbapenem resistance was found in $3.1 \%$ of urosepsis isolates. On the other hand, about $13 \%$ of the non urosepsis $E$. coli isolates carbapenem resistance ( $\mathrm{p}$ value $<0.05$ ). We here with report high prevalence of ESBL production in blood stream $E$. coli isolates. In $E$. coli isolates associated with urosepsis, higher susceptibility towards aminoglycosides and carbapenems was seen. Thus these two groups can serve as empiric antibiotics of choice in a female patient with known predisposing factors for developing a UTI, and presenting with signs and symptoms of urosepsis.

\section{Introduction}

Urosepsis accounts for approximately $25 \%$ of all sepsis cases and may develop from a community or nosocomial acquired urinary tract infection (UTI). Urosepsis refers to the presence of systemic inflammatory response syndrome secondary to a severe infection of the urinary tract, with the risk of multi-organ dysfunction and shock (1). The underlying UTI is almost exclusively a complicated one with involvement of parenchymatous urogenital organs (e.g. kidneys, prostate) (2). The majority of the cases of urosepsis are attributable to gram-negative bacilli. Escherichia coli (E. coli) is the predominant 
organism responsible for a large number of cases of community as well as hospital acquired UTI, across all age groups. E. coli has been reported to cause $50 \%$ to $75 \%$ of cases of urinary tract infection (UTI) with bacteremia, from $(1,3)$. The other organisms implicated in causing urosepsis include Proteus spp., Enterobacter spp, Klebsiella spp. and Pseudomonas spp. Less frequently involved are Gram-positive organisms like Enterococcus spp, Methicillin resistant Staphylococcus aureus and Staphylococcus epidermidis. In this paper we reviewed the clinical and microbiological profile of urosepsis $E$. coli isolates for a year. The primary aim of the study was to analyze the antimicrobial susceptibility profile of $E$. coli isolates causing urosepsis and to compare it with the susceptibility profile of other isolates of $E$. coli from blood stream infection. Whereas the secondary aim was to study the various factors associated with $E$. coli urosepsis cases.

\section{Materials and Methods}

This cross sectional, observational study was carried out in a tertiary care centre in south India. All non-duplicated consecutive isolates of $E$. coli causing blood stream infection for a year were included in the study. Blood culture was performed on the samples with the BacTAlert automated system (bioMe'rieux). The growth positive specimens were Gram stained and subcultured onto Blood agar and MacConkey agar. The gram negative bacilli were identified based on the cultural characteristics and biochemical reactions using preliminary screening media- mannitol motility medium, triple sugar iron agar, indole production and citrate utilization. Antimicrobial susceptibility by Kirby Bauer disk diffusion was performed, as per Clinical and Laboratory Standards Institute (CLSI 2014) guidelines. The E coli isolates were classified into two groups, first group comprising of isolates causing urosepsis and the other including isolates from sepsis without a concomitant or preceding UTI. An isolate was considered as an urosepsis isolate only if the same organism grew in blood culture along with or subsequent to a growth in urine culture, with the two showing the same antimicrobial susceptibility pattern. The susceptibility patterns and multidrug resistance rates of the urosepsis $E$. coli isolates were studied and compared with the nonurosepsis isolates. Relevant demographic and clinical details of the urosepsis patients were obtained from the medical records, and the associated factors were studied.

\section{Results and Discussion}

A total of 689 isolates of $E$. coli were isolated from blood cultures in 2015. Of them 112 were identified to be associated with urosepsis. The mean age of the urosepsis patients was 46.26 years (SD 17.24) and $65.1 \%$ were females. The various demographic and associated factors for the urosepsis cases are mentioned in table 1.

The antibiotic susceptibility rates of the urosepsis isolates were compared with the non-urosepsis isolates and are depicted in table 2 .

\section{Prevalence of multidrug resistance in the two groups}

The prevalence of Extended spectrum beta lactamase production (ESBL), as screened by disc diffusion testing using ceftazidime was almost similar in the two groups, $76 \%$ in the isolates from urosepsis, and $72 \%$ in the other group ( $\mathrm{p}$ value $>0.05$ ).

Carbapenem resistance was found in $3.1 \%$ of urosepsis isolates. On the other hand, about $13 \%$ of the non urosepsis $E$. coli isolates carbapenem resistance ( $\mathrm{p}$ value $<0.05$ ). 
Table.1 Demographic and clinical factors associated with urosepsis $E$. coli isolates

\begin{tabular}{|l|l|}
\hline & Percentage \\
\hline Gender & \\
Male & $34.9 \%$ \\
\hline Female & $65.1 \%$ \\
\hline Age & \\
$\mathbf{0 - 1 6}$ & $5.6 \%$ \\
$\mathbf{1 7 - 4 0}$ & $45.8 \%$ \\
$\mathbf{4 1 - 6 0}$ & $26.3 \%$ \\
$\mathbf{> 6 0}$ & $22.3 \%$ \\
\hline Diabetes & $38.2 \%$ \\
\hline Urinary tract abnormalities & $14 \%$ \\
\hline Post-operative & $6.2 \%$ \\
\hline Post-labour & $7.2 \%$ \\
\hline Pregnancy & $3 \%$ \\
\hline Chemotherapy & $2.9 \%$ \\
\hline & \\
\hline
\end{tabular}

Table.2 Percentage susceptibility of blood stream E. coli isolates (urosepsis vs. others) to various antibiotics

\begin{tabular}{|l|c|c|}
\hline Antibiotic & $\begin{array}{c}\text { Urosepsis causing } \\
\text { E.coli }(\boldsymbol{\%})\end{array}$ & $\begin{array}{c}\text { Other isolates of } \\
\text { E.coli } \mathbf{( \% )}\end{array}$ \\
\hline Ceftazidime & 32.7 & 32.9 \\
\hline Gentamicin & 52.3 & 47.7 \\
\hline Amikacin & 92.1 & 89.89 \\
\hline Netilmycin & 93.6 & $83.2^{*}$ \\
\hline Ciprofloxacin & 23.3 & 23.9 \\
\hline Amoxi-clav & 40 & $\mathrm{NA}$ \\
\hline Cotrimoxazole & 37.2 & $\mathrm{NA}$ \\
\hline Nitrofurantoin & 83.3 & $\mathrm{NA}$ \\
\hline Piperacillin/tazobactam & 60 & 61.1 \\
\hline Cefoperazone/sulbactam & 85.2 & $72.9^{*}$ \\
\hline Meropenem & 98.5 & $85.1^{*}$ \\
\hline Imipenem & 96.5 & $82.9^{*}$ \\
\hline Tigecycline & 99.8 & 98.9 \\
\hline Colistin & 100 & 98.4 \\
\hline *P $<0.05$ & &
\end{tabular}

Infections of the urinary tract are responsible for a large number of cases of secondary healthcare-associated bacteremia. Female gender, older age, urinary catheterization, diabetes mellitus, urinary tract obstruction and pregnancy are known risk factors for developing a urinary tract infection $(4,5)$. In this study, about $65 \%$ of the patients were females. About $46 \%$ of the infections occurred in the age group 17 to 40 , followed by the elderly (26.3\%). Diabetes mellitus was found to be the most common associated 
factor, seen in $38.2 \%$ of the patients. High rates of resistance to ciprofloxacin $(77 \%)$ and trimethoprim-sulfamethoxazole $(65.4 \%)$ were noted. This is consistent with the reports of increasing resistance to these drugs seen in large population based studies(6,7). High rates of prevalence of extended spectrum beta-lactamase producers was seen, which has also been reported in other studies on $E$. coli bacteremia. ESBL rates between $41 \%$ to $76 \%$ have been reported from various parts of the country(8-10). Significantly higher susceptibility to amikacin and netilmycin $(p<0.05)$ was seen in urosepsis isolates, when compared to the non-urosepsis $E$. coli isolates. On the other hand, no significant difference in susceptibility to gentamicin was seen $(p>0.05)$.

Majority of the isolates were resistant to amoxicillin-clavulanic acid, followed by piperacillin-tazobactam, while only $15 \%$ were resistant to cefoperazone-sulbactam. The prevalence of carbapenem resistance was found to be significantly $(\mathrm{P}<0.05)$ low in urosepsis isolates compared to the nonurosepsis isolates. These rates are comparable to the rates reported from other centres. While the multicentric SENTRY study(11) conducted across in 14 hospitals in various Indian cities in 2006-2007 showed an overall resistance of $2.7 \%$ in Enterobacteriaceae isolates, Dutta et al., (12) in their analysis of ten year resistance profile of blood stream infections found $6 \%$ carbapenem resistance in E. coli.

The isolates were largely susceptible to nitrofurantoin. No resistance was seen to colistin and tigecycline.

In conclusion, we here with report high prevalence of ESBL production in blood stream E. coli isolates. In E. coli isolates associated with urosepsis, higher susceptibility towards aminoglycosides and carbapenems was seen. Thus these two groups can serve as empiric antibiotics of choice in a female patient with known predisposing factors for developing a UTI, and presenting with signs and symptoms of urosepsis.

\section{References}

1. Kalra OP, Raizada A. Approach to a Patient with Urosepsis. J Glob Infect Dis. 2009; 1(1):57-63.

2. Wagenlehner FME, Pilatz A, Weidner W. Urosepsis - from the view of the urologist. Int J Antimicrob Agents. 2011 Dec; 38, Supplement:51-7.

3. Al-Hasan MN, Eckel-Passow JE, Baddour LM. Bacteremia complicating gramnegative urinary tract infections: a population-based study. J Infect. 2010 Apr; 60(4): 278-85.

4. Platt R, Polk BF, Murdock B, Rosner B. Risk Factors for Nosocomial Urinary Tract Infection. Am J Epidemiol. 1986 Dec 1; 124(6): 977-85.

5. Ramzan M, Bakhsh S, Salam A, Khan GM, Mustafa G. Risk Factors in Urinary Tract Infection. Gomal J Med Sci [Internet]. 2004 Jun 1 [cited 2015 Jan 27]; 2(1). Available from: http://www.gjms. com.pk/ojs/index.php/gjms/article/view/2 3

6. Al-Hasan MN, Lahr BD, Eckel-Passow JE, Baddour LM. Antimicrobial resistance trends of Escherichia coli bloodstream isolates: a population-based study, 19982007. J Antimicrob Chemother. 2009 Jul; 64(1):169-74.

7. Laupland KB, Gregson DB, Church DL, Ross T, Pitout JDD. Incidence, risk factors and outcomes of Escherichia coli bloodstream infections in a large Canadian region. Clin Microbiol Infect Off Publ Eur Soc Clin Microbiol Infect Dis. 2008 Nov; 14(11):1041-7.

8. Nasa P, Juneja D, Singh O, Dang R, Singh A. An observational study on bloodstream 
extended-spectrum beta-lactamase infection in critical care unit: incidence, risk factors and its impact on outcome. Eur J Intern Med. 2012 Mar;23(2):192-5.

9. Taneja J, Mishra B, Thakur A, Dogra V, Loomba P. Nosocomial blood-stream infections from extended-spectrum-betalactamase-producing Escherichia coli and Klebsiella pneumonia from GB Pant Hospital, New Delhi. J Infect Dev Ctries. 2010 Aug;4(8):517-20.

10. Shashwati N, Kiran T, Dhanvijay AG. Study of extended spectrum $\beta$-lactamase producing Enterobacteriaceae and antibiotic coresistance in a tertiary care teaching hospital. J Nat Sci Biol Med. 2014 Jan;5(1):30-5.
11. Castanheira M, Deshpande LM, Mathai D, Bell JM, Jones RN, Mendes RE. Early Dissemination of NDM-1- and OXA-181Producing Enterobacteriaceae in Indian Hospitals: Report from the SENTRY Antimicrobial Surveillance Program, 2006-2007. Antimicrob Agents Chemother. 2011 Mar;55(3):1274-8.

12. Datta S, Wattal C, Goel N, Oberoi JK, Raveendran R, Prasad KJ. A ten year analysis of multi-drug resistant blood stream infections caused by Escherichia coli and Klebsiella pneumoniae in a tertiary care hospital. Indian J Med Res. 2012 Jun;135(6):907-12.

\section{How to cite this article:}

Pragya Agarwala, Archa Sharma and Kamal Sharma. 2018. Antimicrobial Susceptibility Profile of E. coli Isolates Causing Urosepsis: Single Centre Experience. Int.J.Curr.Microbiol.App.Sci. 7(05): 2585-2589. doi: https://doi.org/10.20546/ijcmas.2018.705.298 